\title{
Structural dynamics of photochemical reactions probed by time-resolved photoelectron spectroscopy using high harmonic pulses
}

\author{
Ryo likubo, ${ }^{a}$ Taro Sekikawa, ${ }^{* a}$ Yu Harabuchi ${ }^{\mathrm{b}}$ and Tetsuya Taketsugu ${ }^{\mathrm{b}}$
}

Received 31st March 2016, Accepted 31st May 2016

DOI: $10.1039 / \mathrm{c} 6 \mathrm{fd} 00063 \mathrm{k}$

Femtosecond ring-opening dynamics of 1,3-cyclohexadiene (CHD) in gas phase upon two-photon excitation at $400 \mathrm{~nm}(=3.1 \mathrm{eV})$ was investigated by time-resolved photoelectron spectroscopy using $42 \mathrm{~nm}(=29.5 \mathrm{eV})$ high harmonic photons probing the dynamics of the lower-lying occupied molecular orbitals (MOs), which are the fingerprints of the molecular structure. After $500 \mathrm{fs}$, the photoelectron intensity of the $\mathrm{MO}$ constituting the $\mathrm{C}=\mathrm{C}$ sigma bond $\left(\sigma_{\mathrm{C}=\mathrm{C}}\right)$ of $\mathrm{CHD}$ was enhanced, while that of the $\mathrm{MO}$ forming the $\mathrm{C}-\mathrm{C}$ sigma bond $\left(\sigma_{\mathrm{CC}}\right)$ of $\mathrm{CHD}$ was decreased. The changes in the photoelectron spectra suggest that the ring of $\mathrm{CHD}$ opens to form a 1,3,5-hexatriene (HT) after 500 fs. The dynamics of the $\sigma_{\mathrm{C}=\mathrm{C}}$ and $\sigma_{\mathrm{CC}}$ bands between 200 and 500 fs reflects the ring deformation to a conical intersection between the $2^{1} \mathrm{~A}$ and $1^{1} \mathrm{~A}$ potential energy surfaces prior to the ring-opening reaction.

\section{Introduction}

Electrocyclic reactions play important roles in biological activities ${ }^{\mathbf{1}}$ and in photoelectric functions. ${ }^{2}$ The stereospecificity of electrocyclic ring-opening and ring-closing reactions is well understood by the Woodward-Hoffmann rules ${ }^{3}$ and the dynamics of the electrocyclic reactions has also been investigated experimentally. In particular, 1,3-cyclohexadiene (CHD) is of interest, ${ }^{4,5}$ because the ring-opening reaction of $\mathrm{CHD}$ to $1,3,5$-hexatriene (HT) is the key reaction in the synthesis of vitamin $\mathrm{D}^{6-8}$ and in photochromic materials. ${ }^{2}$ CHD provides us a unique opportunity to investigate the ring-opening dynamics of biological and functional molecules in a simplified manner.

To gain insight into the intrinsic ring-opening dynamics without the interaction with solvent, a gas-phase experiment is suitable. The ring-opening

${ }^{a}$ Department of Applied Physics, Faculty of Engineering, Hokkaido University, Kita 13 Nishi 8, Kita-ku, Sapporo 060-8628, Japan. E-mail: sekikawa@eng.hokudai.ac.jp

${ }^{b}$ Department of Chemistry, Faculty of Science, Hokkaido University, Kita 10 Nishi 8, Kita-ku, Sapporo 060o810, Japan 
dynamics of CHD in gas phase has been extensively investigated by ionization mass and photoelectron spectroscopy, ${ }^{9-17}$ electron diffraction, ${ }^{18}$ X-ray scattering, ${ }^{19}$ theoretical calculations, ${ }^{20-24}$ and so forth. These investigations focus on the relaxation processes from the $1^{1} \mathrm{~B}$ state. The configurational coordinate model shown in Fig. 1 is used to understand the processes: upon photoexcitation to $1^{1} \mathrm{~B}$, the nuclear wavepacket propagates to the $2^{1} \mathrm{~A}$ state via a conical intersection (CI). At the second CI with the $1^{1} \mathrm{~A}$ state on the $2^{1} \mathrm{~A}$ potential energy surface, it bifurcates into CHD and HT in the ground state. Experimentally, it was found that the internal conversion from $1^{1} \mathrm{~B}$ to $2^{1} \mathrm{~A}$ took 56 fs by the transient ionization experiment. ${ }^{12}$ Time-resolved photoelectron spectroscopy (TRPES) using ultraviolet (UV) light ${ }^{17}$ showed that the lifetime of $1^{1} \mathrm{~B}$ was $30 \mathrm{fs}$ and that HT appeared at $142 \mathrm{fs}$ after excitation. This is consistent with other works. ${ }^{11,12}$ New light sources were applied to the time-resolved spectroscopy of CHD recently; ${ }^{16}$ TRPES probed by $14 \mathrm{eV}$ light gave the similar lifetimes of 70 and 60 fs for $1^{1} \mathrm{~B}$ and $2^{1} \mathrm{~A}$, respectively, to those obtained by UV light. Structural dynamics observed by X-ray scattering using a free-electron laser (FEL) showed that the bond of CHD broke as the terminal carbon atoms moved perpendicular to the molecular plane. ${ }^{19}$ Electron diffraction ${ }^{18}$ showed that the CHD ring cleaved on the picosecond timescale including the isomerization processes of HT.

Here, we investigated the ring-opening dynamics pumped by two photons of $400 \mathrm{~nm}(=3.1 \mathrm{eV})$ light and probed it by TRPES using high harmonic pulses with a photon energy of $29.5 \mathrm{eV}$. The present experimental scheme has two features in comparison with the previous studies: first, $3 p$ Rydberg states, found by the resonant-enhanced multiphoton ionization (REMPI), ${ }^{20}$ were excited by the twophoton absorption process, while the $1^{1} \mathrm{~B}$ state corresponding to $\pi \pi^{*}$ transition was excited in the previous works. ${ }^{9-17}$ According to ref. 11, the ring opening was observed even at zero delay time under the two-photon excitation by $400 \mathrm{~nm}$ photons. This suggests that there are some very fast nonradiative processes from the Rydberg states. Therefore, the ring-opening dynamics might be different from the case of $\pi \pi^{*}$ excitation.

Second, TRPES using high harmonic pulses enables us to take a snapshot of the molecular orbitals (MOs) under an assumption of Koopmans'

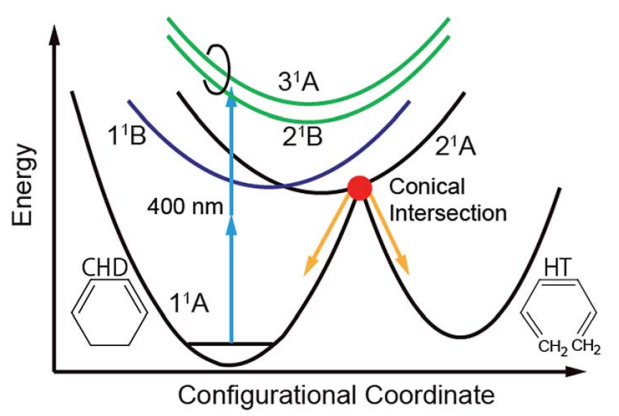

Fig. 1 Configurational coordinate model of the relaxation and the ring-opening processes in 1,3-cyclohexadiene (CHD) pumped by two-photon absorption of $400 \mathrm{~nm}$ photons. The arrows at the conical intersection indicate the bifurcation of the relaxation pathway at the conical intersection, leading to CHD or 1,3,5-hexatriene (HT). 
theorem..$^{25}$ The usage of ultrashort extreme ultraviolet (XUV) pulses by high harmonic generation enables us to probe not only the highest occupied MO (HOMO) but also lower-lying occupied MOs. ${ }^{26-30}$ Lower-lying occupied MOs can be the fingerprints of the molecular structure, because each MO can be associated with each bonding nature, e.g. single bond, double bond, conjugated double bond, and so forth. ${ }^{31}$ The dynamics particular to each MO reflects the local conformational changes of the molecule. Using high harmonic pulses, we can gain insight into both electronic structures and molecular structures, providing us a new perspective on the chemical dynamics. Recently, we observed the ultrafast relaxation of the excited 1,3butadiene $^{30}$ and the $\mathrm{C}-\mathrm{C}$ bond breaking dynamics of 1,2 -butadiene ${ }^{32}$ by TRPES using high harmonic pulses.

\section{Experimental}

The experimental setup is shown in Fig. 2. We used a Ti:sapphire laser system delivering $800 \mathrm{~nm}, 1.1 \mathrm{~mJ}, 30 \mathrm{fs}$ pulses at a repetition rate of $1 \mathrm{kHz}$ for TRPES. After the second harmonic $(=3.1 \mathrm{eV})$ generation by a $\mathrm{LiB}_{3} \mathrm{O}_{5}(\mathrm{LBO})$ crystal (500 $\mu \mathrm{m}$ thickness) for the pump pulses with a pulse energy of $40 \mu \mathrm{J}$, the remaining fundamental pulses were focused into a Kr pulse gas jet for high harmonic generation. The 19th harmonic $(=29.5 \mathrm{eV})$ for the probe was selected among many harmonics by a time-delay compensated monochromator (TDCM), ${ }^{33-37}$ enabling the single harmonic selection with the time duration preserved. A TDCM consists of a pair of toroidal gratings and a slit: the first grating and a slit select the harmonic order and the second grating compensates for the pulse front tilt caused by the diffraction on the first grating. The shortest pulse duration of the selected high harmonics achieved in our system was $11 \mathrm{fs} .{ }^{35}$ The pump intensity was reduced to $19 \mu \mathrm{J}$ by the attenuator composed of a pair of thin film polarizers and a half-wave plate. Both the pump and probe pulses were focused into the sample gas with a polarization angle of $55^{\circ}$ to avoid the time dependence induced by molecular rotation. The photon flux on target was $\sim 10^{9}$ photons per s, corresponding to a pulse energy of 5 pJ. ${ }^{37} \mathrm{CHD}$ was purchased from Sigma-Aldrich and was used without any further purification. The sample gas, evaporated at room temperature, was guided to the entrance of a magnetic

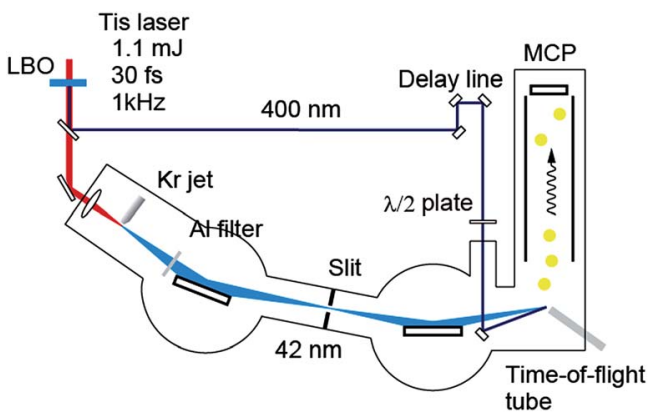

Fig. 2 Experimental setup for time-resolved photoelectron spectroscopy using high harmonic pulses. Tis is Ti:sapphire. 
bottle time-of-flight photoelectron spectrometer through a copper pipe. A retardation electric field was applied in the flight tube to improve the spectral resolution of the spectrometer. Hence, the spectral resolution was determined by the spectral width of the XUV light $(200 \mathrm{meV})$. The transient photoelectron spectra were recorded by changing the optical delay between the pump and probe pulses. The temporal evolution of the photoelectrons ejected by the twophoton ionization of $\mathrm{Kr}$ gas by pump and probe pulses, i.e. the cross-correlation function, ${ }^{38,39}$ was employed as the response function of the system, of which the width $\tau_{\text {FWHM }}$ was $120 \mathrm{fs}$. Since we have already optimized the diffraction angle of the grating in the TDCM ${ }^{35}$ the temporal width was mainly limited by the pump pulse duration.

\section{Computational details}

To understand the photoelectron spectra, the ionization energies of CHD and the isomers of HT were calculated from the orbital energies obtained by longrange corrected density functional theory (LC-DFT) with LC-BOP/cc-pVDZ using the GAMESS-package $e^{25,40}$ under an assumption of Koopmans' theorem. Also, to investigate the origin of the transient states, the vertical excitation energies and ionization energies were calculated at the Franck-Condon (FC) structure of CHD by 2nd-order multi-reference perturbation theory (CASPT2) with Dunning's basis sets of double-zeta-quality plus Rydberg functions, ${ }^{41}$ using MOLPRO2010. ${ }^{42}$ Two sets of $\pi$ and $\pi^{*}\left(12 \mathrm{a}, 10 \mathrm{~b}, \pi^{*}\right.$-a, $\pi^{*}$-b) orbitals and four Rydberg orbitals $\left(3 \mathrm{~s}, 3 \mathrm{p}_{x}, 3 \mathrm{p}_{y}, 3 \mathrm{p}_{z}\right)$ were included in the active space of the reference state-averaged complete active space self-consistent field (SA-CASSCF) wavefunction.
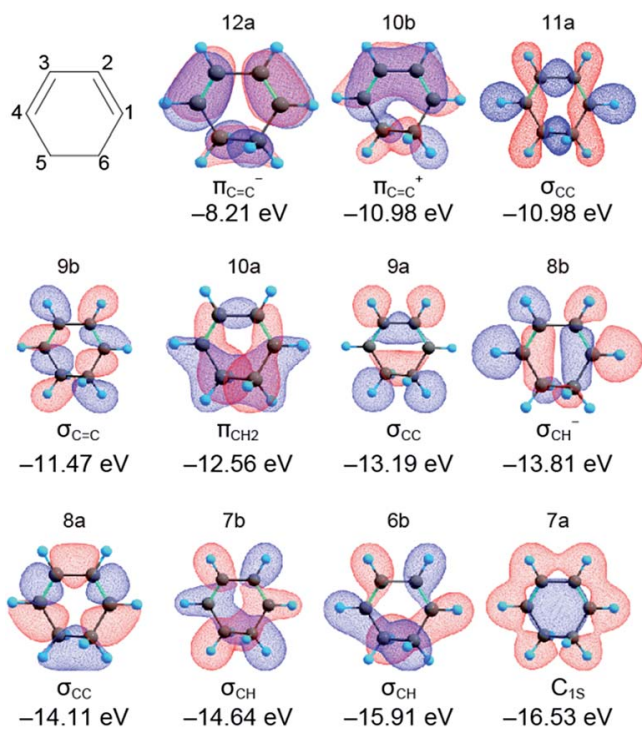

Fig. 3 Structural formula and molecular orbitals of 1,3-cyclohexadiene calculated the LC-BOP/cc-pVDZ level. The character and orbital energy of each MO are also indicated. 


\section{Results and discussion}

\section{Molecular orbitals}

The orbital energies and the MO shapes of CHD are summarized in Fig. 3. The orbital energies are in good agreement with the experimental ionization energies shown later, and thus, Koopmans' theorem is applicable to CHD. Each MO has a different spatial distribution and the character of the MO is named to signify the distribution in reference to ref. 31. The MO distribution is not directly connected with the classical chemical bond used in the structural formula. However, it is worth mentioning that the MOs with $-12.56,-13.19$, and $-14.11 \mathrm{eV}$ have a high density at the C5-C6 bond that cleaves upon photoexcitation. Thus, we expect that the intensities of photoelectrons or the ionization energies of these MOs are changed by the C5-C6 bond cleavage. These MOs can be the fingerprints of the ring structure of CHD.

\section{Excited states}

The calculated and experimental absorption energies of CHD are shown in Table 1. The 3p Rydberg states experimentally have the transition energies of 6.03 and $6.05 \mathrm{eV}$ (ref. 20) and the absorption spectrum has sharp peaks around $6.2 \mathrm{eV},{ }^{43}$ which is a feature of Rydberg states. Since the point group of CHD is $C_{2}$, these states are both one- and two-photon transition allowed. Therefore, the excited states by two-photon absorption of $3.1 \mathrm{eV}$ photons were mainly $3 \mathrm{p}$ Rydberg states in this experiment. The three-photon energy of the pump laser is larger than the ionization energy of $\mathrm{CHD}(=8.25 \mathrm{eV})^{31}$ and the second ionization energy should be much larger than the first ionization energy due to the Coulomb interaction. Hence, the photoelectrons from the cations would appear in a totally different spectral region from the neutral species. In this work, therefore, we eliminate the possibility that the dynamics of cations were observed.

Table 1 The calculated and experimental excitation energies [eV] for the neutral and cationic species of $\mathrm{CHD}$. All values are relative to the ground state energy of $\mathrm{CHD}$

\begin{tabular}{lllll}
\hline & State & CASPT2 $^{a}$ & CASPT2 $^{b}$ & $\exp ^{b}$ \\
\hline \multirow{2}{*}{ Neutral } & $1^{1} \mathrm{~B}\left(12 \mathrm{a} \rightarrow \pi^{*}\right.$-b) & 4.88 & 4.72 & 4.94 \\
& $2^{1} \mathrm{~A}(12 \mathrm{a} \rightarrow 3 \mathrm{~s})$ & 5.41 & 5.49 & 5.39 \\
& $2^{1} \mathrm{~B}\left(12 \mathrm{a} \rightarrow 3 \mathrm{p}_{x}\right)$ & 5.94 & 5.98 & 6.03 \\
& $3^{1} \mathrm{~A}\left(12 \mathrm{a} \rightarrow 3 \mathrm{p}_{z}\right)$ & 6.03 & 6.12 & \\
Cation & $3^{1} \mathrm{~B}\left(12 \mathrm{a} \rightarrow 3 \mathrm{p}_{y}\right)$ & 6.05 & 6.04 & \\
& $1^{2} \mathrm{~A}(\mathrm{GS})$ & 8.09 & & \\
& $1^{2} \mathrm{~B}(10 \mathrm{~b} \rightarrow 12 \mathrm{a})$ & 10.53 & & \\
$2^{2} \mathrm{~B}\left(12 \mathrm{a} \rightarrow \pi^{*}-\mathrm{b}\right)$ & 11.78 & & \\
& $2^{2} \mathrm{~A}\left(12 \mathrm{a} \rightarrow \pi^{*}\right.$-a) & 13.86 & & \\
$3^{2} \mathrm{~A}(12 \mathrm{a} \rightarrow 3 \mathrm{~s})$ & 16.00 & & \\
$3^{2} \mathrm{~B}\left(12 \mathrm{a} \rightarrow 3 \mathrm{p}_{x}\right)$ & 17.03 & &
\end{tabular}




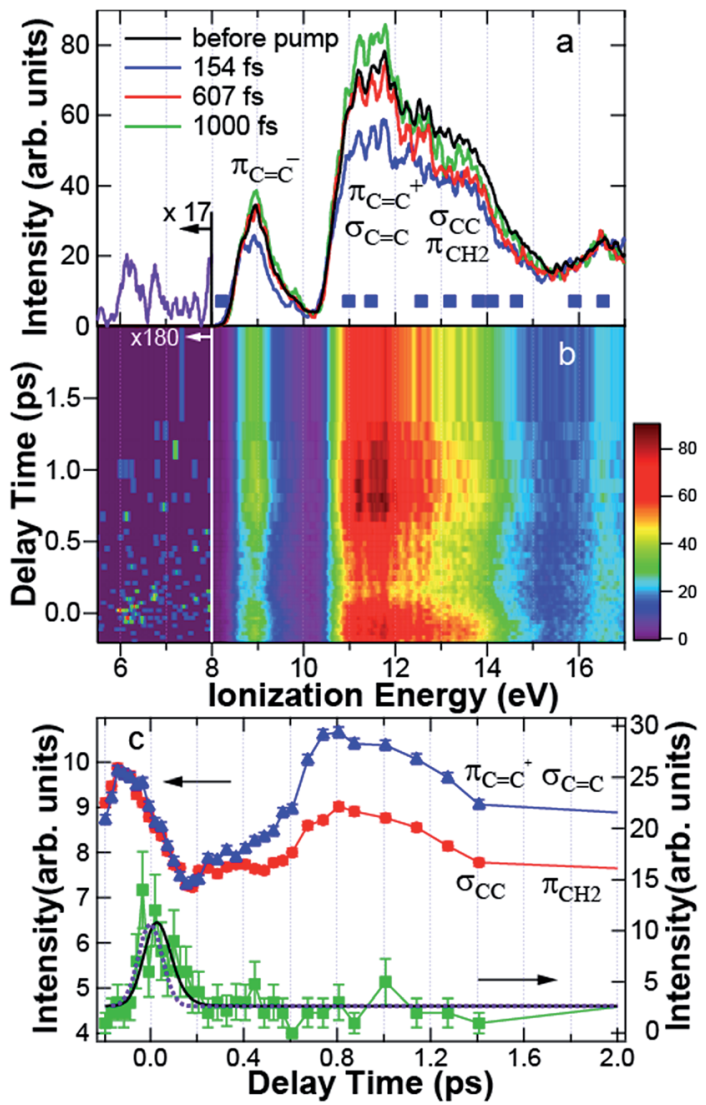

Fig. 4 (a) Photoelectron spectrum before the pump (black) and the spectra at 154 fs (blue), $607 \mathrm{fs}$ (red), and $1000 \mathrm{fs}$ (green). The solid squares indicate the theoretical ionization energies of 1,3-cyclohexadiene. In the assignment to molecular orbitals, notations are taken from Fig. 3. The spectrum of the transient band integrated between -140 and $250 \mathrm{fs}$ is shown by the purple line below $8 \mathrm{eV}$. (b) Photoelectron spectrogram between -0.2 and 2 ps. (c) Time dependence of the intensity of $\pi_{\mathrm{C}=\mathrm{C}^{+}}$and $\sigma_{\mathrm{C}=\mathrm{C}}$ (blue triangle), that of $\sigma_{\mathrm{CC}}$ and $\pi_{\mathrm{CH}_{2}}$ (red circle), and the transient band (green square) integrated between 11.14 and $11.93 \mathrm{eV}, 12.20$ and $14.00 \mathrm{eV}$, and 5.69 and $7.75 \mathrm{eV}$, respectively. The blue, red, and green solid lines are a guide for the eye. The black solid and purple dotted lines are the results fitted to the experimental data and the response function of the system, respectively.

\section{Time-resolved photoelectron spectra}

In Fig. $4 \mathrm{a}$, the photoelectron spectrum before the pump is shown by the black line, obtained by averaging the spectra at 5 negative delay times between $-200 \mathrm{fs}$ and -86 fs. Two broad photoelectron bands between 10.5 and $12 \mathrm{eV}$ and between 12 and $14 \mathrm{eV}$ are clearly shown. The broad spectrum is due to the spectral width of the probe light. The theoretical ionization energies of CHD indicated by the solid squares are found within the observed spectrum. In particular, the photoelectron band between 12 and $14 \mathrm{eV}$ includes $\sigma_{\mathrm{CC}}$ at $13.19 \mathrm{eV}$ and $\pi_{\mathrm{CH}_{2}}$ at $12.56 \mathrm{eV}$ constituting the C5-C6 bond, while the band between 10.5 and $12 \mathrm{eV}$ is contributed by $\pi_{\mathrm{C}=\mathrm{C}^{+}}$at $10.98 \mathrm{eV}$ and $\sigma_{\mathrm{C}=\mathrm{C}}$ at $11.47 \mathrm{eV}$ forming the $\mathrm{C}=\mathrm{C}$ bond. To 
characterize each photoelectron band based on the MOs, we denote hereafter the band between 12 and $14 \mathrm{eV}$ as the $\mathrm{C}-\mathrm{C}$ band, and the band between 10.5 and $12 \mathrm{eV}$ as the $\mathrm{C}=\mathrm{C}$ band.

Fig. 4a also shows the time-resolved photoelectron spectra at 154 fs (blue), 607 fs (red), and 1000 fs (green) after pump. Fig. 4b shows the photoelectron spectrogram between -200 and 2000 fs. Here, we would like to raise the following two features in Fig. $4 \mathrm{a}$ and b. First, the dynamics of the $\mathrm{C}-\mathrm{C}$ and $\mathrm{C}=\mathrm{C}$ bands are different to each other: the photoelectron yield was reduced uniformly just after excitation. However, the intensity of the $\mathrm{C}=\mathrm{C}$ band was almost recovered at 607 $\mathrm{fs}$, while that of the $\mathrm{C}-\mathrm{C}$ band was still reduced. At $1000 \mathrm{fs}$, the $\mathrm{C}=\mathrm{C}$ band was even enhanced, although the $\mathrm{C}-\mathrm{C}$ band intensity was still reduced. Second, Fig. $4 \mathrm{~b}$ shows that a transient state appeared around $6-7 \mathrm{eV}$ at $0 \mathrm{fs}$. The spectrum of the transient band below $8 \mathrm{eV}$ integrated between -140 and $250 \mathrm{fs}$ is shown in Fig. $4 \mathrm{a}$ by the purple line. The transient state has a broad spectrum almost reaching to the HOMO band.

To see these MO-dependent dynamics, the intensities of the $\mathrm{C}=\mathrm{C}, \mathrm{C}-\mathrm{C}$, and the transient bands integrated between 11.14 and $11.93 \mathrm{eV}, 12$ and $14 \mathrm{eV}$, and 5.69 and $7.75 \mathrm{eV}$, respectively, were plotted as a function of delay time between -0.2 and 2 ps in Fig. 4c. Here, the intensities of the $\mathrm{C}-\mathrm{C}$ band and the $\mathrm{C}=\mathrm{C}$ band are magnified to be on the same scale to verify that their dynamics are quite similar between -200 and 150 fs. The error bars show the statistical errors in the counting experiments. The time dependence of the transient band was fitted by the least squares method to a single exponential function $I(t)=\exp (-t / \tau)$ convoluted with the correlation function of the system $R(t)=\exp \{-4 \ln 2(t)$ $\left.\left.\tau_{\text {FWHM }}\right)^{2}\right\}$, where $t, \tau$, and $\tau_{\text {FWHM }}$ are the time, decay time, and temporal width of $R(t)$, respectively. The fitting function $f(t)$ is expressed as.

$$
f(t)=A \int_{-\infty}^{t} R\left(t^{\prime}\right) I\left(t-t^{\prime}\right) \mathrm{d} t^{\prime}+B
$$

where $A$ and $B$ are the amplitude and the background constant, respectively. The fitting parameters were $A, B$, and $\tau$. $\tau_{\text {FWHM }}$ was set to 120 fs. $R(t)$ and the fitting result are shown by the dotted purple line and the solid black line, respectively, in Fig. 4c. The lifetime of the transient band $\tau$ from the fitting was $37 \pm 13 \mathrm{fs}$.

\section{Ring-opening dynamics}

Fig. $4 \mathrm{c}$ shows that, after $150 \mathrm{fs}$, the $\mathrm{C}=\mathrm{C}$ band increased gradually until $\sim 500 \mathrm{fs}$. Then, the intensity was enhanced up to $800 \mathrm{fs}$, while the $\mathrm{C}-\mathrm{C}$ band was constant between 200 and $500 \mathrm{fs}$ and was also enhanced after $500 \mathrm{fs}$. However, the intensity of the $\mathrm{C}-\mathrm{C}$ band was not fully recovered in the spectrum before the pump. Because the $\mathrm{C} 5-\mathrm{C} 6$ bond mainly comes from the $\mathrm{C}-\mathrm{C}$ band, the decrease in the relative intensity of the $\mathrm{C}-\mathrm{C}$ band to the $\mathrm{C}=\mathrm{C}$ band can be associated with the cleavage of the $\mathrm{C}-\mathrm{C}$ bond.

To find the intermediates and products of the ring-opening reaction, the difference time-resolved spectra from the photoelectron spectrum before the pump are shown at delay times of 154, 607, and 1000 fs in Fig. 5b. Here, we consider three isomers of HT, all-cis-hexatriene (cZc-HT), mono-s-cis-Z-hexatriene (cZt-HT), and di-s-trans-Z-hexatriene (tZt-HT). Their chemical formulas are shown in Fig. 6 . The ionization energies of these isomers, calculated by the same method 


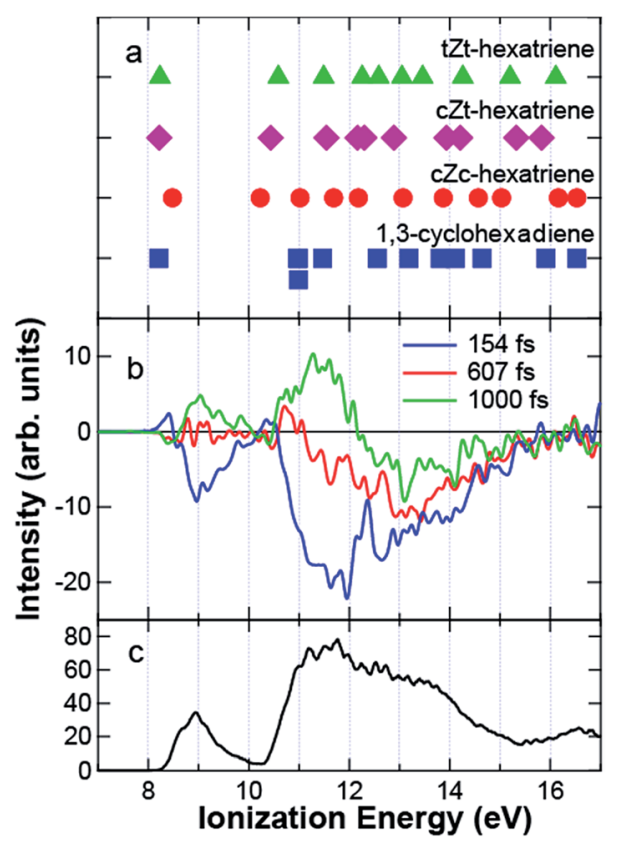

Fig. 5 (a) Ionization energies of tZt-hexatriene (triangle), cZt-hexatriene (diamond), cZchexatriene (circle), and 1,3-cyclohexadiene (CHD) (square). (b) Difference photoelectron spectra from the averaged photoelectron spectrum in the negative delay time. (c) Photoelectron spectrum of CHD without optical pump.

as those of CHD, are shown in Fig. 5a together with those of CHD. Fig. 5c shows the photoelectron spectrum of CHD without the pump for comparison. Although we found that the $\mathrm{C}=\mathrm{C}$ band was enhanced in Fig. $4 \mathrm{c}$, Fig. $5 \mathrm{~b}$ shows that the temporal evolution of the $\mathrm{C}=\mathrm{C}$ band was not uniform: the photoelectron band with a lower ionization energy than $11 \mathrm{eV}$ was enhanced faster than the energetically higher region even within the $\mathrm{C}=\mathrm{C}$ band. As a result, the positive signal appeared below $11 \mathrm{eV}$ at 607 fs in Fig. 5b. This suggests that an MO with an ionization energy lower than $11 \mathrm{eV}$ appeared by ring opening. The theoretical ionization energies of HTs shown in Fig. 5a predict that HTs have smaller ionization energies around $10.2 \mathrm{eV}$ than that of the $\pi_{\mathrm{C}=\mathrm{C}^{+}}$of CHD. The theoretical calculation also predicts the energy shift of the HOMO of cZc-HT to the higher ionization energy, which was experimentally observed at $1000 \mathrm{fs}$ in Fig. 5b. Therefore, we attribute the MO lower than $11 \mathrm{eV}$ observed at 607 fs to $\pi_{\mathrm{C}=\mathrm{C}}$ of cZcHT produced just after the ring opening reaction. At almost the same delay time,

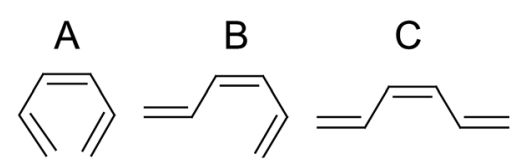

Fig. 6 Chemical formulas of hexatriene isomers. (A) cZc-hexatriene, (B) cZt-hexatriene, and (C) tZt-hexatriene. 
we also observed the enhancement of the $\mathrm{C}-\mathrm{C}$ band in Fig. 4c, suggesting a drastic change in the electronic structure, i.e. ring opening, of the molecule. Thus, we conclude that the ring starts to open at $\sim 500 \mathrm{fs}$.

The enhancement of the $\mathrm{C}=\mathrm{C}$ band compared to the $\mathrm{C}-\mathrm{C}$ band upon ring opening is qualitatively understood as the change in the electronic structure from CHD to cZc-HT. If the ionization cross section of each MO is almost the same, the photoelectron intensity is proportional to the number of MOs involved in a selected region: $\mathrm{CHD}$ has three MOs in the $\mathrm{C}=\mathrm{C}$ band between 10 and $12.2 \mathrm{eV}$, while cZc-HT has four shown in Fig. 5a. Thus, the $\mathrm{C}=\mathrm{C}$ band is expected to be enhanced by ring opening. On the other hand, CHD has three MOs in the C-C band between 12.2 and $14.0 \mathrm{eV}$, while cZc-HT has two, also explaining the decrease in the $\mathrm{C}-\mathrm{C}$ band by ring opening. The enhancement of the $\mathrm{C}=\mathrm{C}$ and $\mathrm{C}-\mathrm{C}$ bands ended at $800 \mathrm{fs}$ and then, their band intensities began to be reduced till 1.4 ps. The relative intensity of the $\mathrm{C}-\mathrm{C}$ band to the $\mathrm{C}=\mathrm{C}$ band remained smaller after $800 \mathrm{fs}$, suggesting that the molecule stayed at the HT structures. Therefore, the decrease in the photoelectron intensities of both the $\mathrm{C}-\mathrm{C}$ and the $\mathrm{C}=\mathrm{C}$ bands after 800 fs are attributable to subsequent isomerization to cZt-HT or to tZt-HT.

Fig. 7 shows the long-term measurement of the $\mathrm{C}=\mathrm{C}$ and $\mathrm{C}-\mathrm{C}$ bands. After 7 ps, the intensities of both bands increased but the $\mathrm{C}=\mathrm{C}$ band was more enhanced. Then, at $40 \mathrm{ps}$, the $\mathrm{C}=\mathrm{C}$ band intensity decreased. Fig. $5 \mathrm{a}$ shows that the changes in the ionization energies among the isomers of HT are not so significant. Thus, the assignment of the molecular structure is not conclusive. However, the structural dynamics observed by the electron diffraction revealed the picosecond dynamics and the coexistence of HT isomers. ${ }^{18}$ After the ring opening, the rotational inertia around the $\mathrm{C}-\mathrm{C}$ bond should induce the isomerization of HT. As a result, the long-term dynamics shown in Fig. 7 is attributed to the isomerization among HTs.

TRPES using ultraviolet light also suggested that HTs have the higher vibrational temperature. ${ }^{17}$ According to ref. 17, the deconvoluted photoelectron spectrum of HT extended to the lower ionization energies. This suggests that the isomers of HTs coexist and that anharmonic vibrations broaden the spectrum.

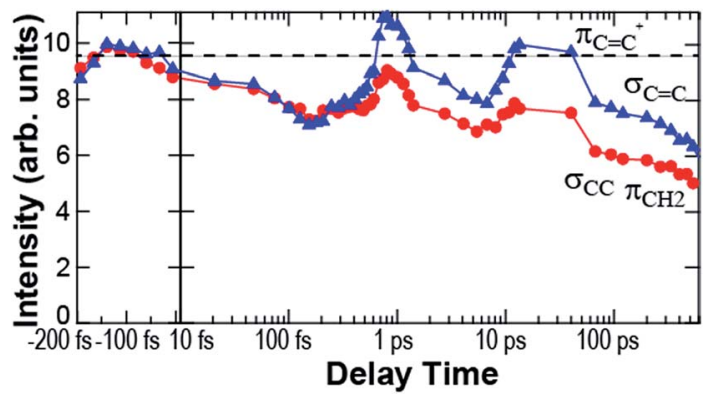

Fig. 7 Time dependence of the intensity of $\pi_{\mathrm{C}=\mathrm{C}^{+}}$and $\sigma_{\mathrm{C}=\mathrm{C}}$ (blue triangle) and that of $\sigma_{\mathrm{CC}}$ and $\pi_{\mathrm{CH}_{2}}$ (red circle) integrated between 11.14 and $11.93 \mathrm{eV}$ and 12.20 and $14.00 \mathrm{eV}$, respectively. Delay times are presented on a logarithmic and on a linear scale in the positive and negative regions, respectively. The blue and red solid lines are a guide for the eye. The dotted line indicates the intensity of $\pi_{\mathrm{C}=\mathrm{C}^{+}}$and $\sigma_{\mathrm{C}=\mathrm{C}}$ in the averaged spectrum. 


\section{Dynamics of transient states}

Now, we would like to discuss the processes prior to the ring opening. Just after excitation, we observed the transient state around 6-7 eV. We attribute this transient state to the FC states of $3 p$ Rydberg states, which relax to the lower-lying excited states through CIs with a time constant of $37 \pm 13 \mathrm{fs}$. The corresponding relaxation dynamics of the $\mathrm{C}=\mathrm{C}$ and $\mathrm{C}-\mathrm{C}$ bands was also observed: upon photoexcitation, both the $\mathrm{C}=\mathrm{C}$ and $\mathrm{C}-\mathrm{C}$ band intensities decreased with the response function of the system, owing to the different ionization cross section of the ground state or FC factors from those of the excited states, and then, the slight increment was observed at $200 \mathrm{fs}$ in Fig. 4c. The relaxation of the transient state took place simultaneously with these increments of the $\mathrm{C}=\mathrm{C}$ and $\mathrm{C}-\mathrm{C}$ bands, indicating the relaxation to the lowerlying excited states. The broad spectral width of the transient state over $2 \mathrm{eV}$ shown in Fig. 4a suggests that the transient band is composed of more than a few states.

As shown in Table 1, the present calculations well reproduced the experimental and previously calculated excitation energies for neutral CHD. ${ }^{20}$ The first ionization energy was calculated as $8.09 \mathrm{eV}$ which is consistent with the HOMO energy from LC-BOP calculations $(8.21 \mathrm{eV})$. According to the relative energy values in Table 1, the ionization energies from the $3^{1} \mathrm{~A}$ state of the neutral species to the cationic states, $2^{2} \mathrm{~B}\left(12 \mathrm{a} \rightarrow \pi^{*}\right.$-b) and $2^{2} \mathrm{~A}\left(12 \mathrm{a} \rightarrow \pi^{*}\right.$-a), are 5.75 and $7.83 \mathrm{eV}$, respectively. Thus, the observed transient photoelectron bands could be assigned to these two states.

We did not detect any signals via the transition from the $3 p$ Rydberg states of the neutral species to the $1^{2} \mathrm{~A}$ and $1^{2} \mathrm{~B}$ states of the cation, which are expected to appear around 2.06 and $4.50 \mathrm{eV}$, respectively. We think that this is due to the lower collection efficiency for the faster photoelectrons of the magnetic bottle spectrometer, because the faster electrons are not well guided to the detector due to the larger Larmor radius of the electron in the magnetic field. ${ }^{\mathbf{4}}$

\section{Dynamics on the $2^{1} \mathrm{~A}$ potential energy surface}

After the relaxation to the $2^{1} \mathrm{~A}$ or $1^{1} \mathrm{~B}$ state, the temporal evolution of the $\mathrm{C}=\mathrm{C}$ and $\mathrm{C}-\mathrm{C}$ bands observed between 200 and 500 fs should reflect the dynamics in the $2^{1} \mathrm{~A}$ or $1^{1} \mathrm{~B}$ excited states. Tamura and coworkers investigated the quantum dynamics of CHD on the potential energy surfaces, calculated by a multireference configuration interaction method, after excitation to the $1^{1} \mathrm{~B}$ state. ${ }^{23}$ According to this report, the wavepacket decays from the FC state to $2^{1} \mathrm{~A}$ through a CI on the $1^{1} \mathrm{~B}-2^{1} \mathrm{~A}$ seam line. In the $2^{1} \mathrm{~A}$ state, the ring structure is distorted firstly to the open-ring, then to the closed-ring, and finally to the five-membered ring ( $5 \mathrm{MR}$ ) structures. Since a CI with the $1^{1} \mathrm{~A}$ state is located close to $5 \mathrm{MR}$, the molecular structure bifurcates into CHD or cZc-HT in the ground state. Due to dynamics in the $2{ }^{1} \mathrm{~A}$ state, it is predicted that the appearance of cZc-HT has about $180 \mathrm{fs}$ time lag behind photoexcitation. In the present experiment, CHD was excited to the $3 p$ Rydberg states. Therefore, the relaxation pathway from the FC structure on the potential energy surface does not necessarily lead to the $1^{1} \mathrm{~B}$ state and the experimental results might not be directly comparable to the theoretical work by Tamura et al. ${ }^{23}$ However, the time-resolved photoelectron spectra show that the CHD ring opened not just after photoexcitation but after 
500 fs. We would like to ascribe this delayed ring-opening reaction to the ringdeformation dynamics in the $2^{1} \mathrm{~A}$ state after the relaxation from $3 p$ Rydberg states. Still, we do not have any idea why only the $\mathrm{C}=\mathrm{C}$ band intensity increases gradually during the ring deformation. We suspect that the ionization cross sections of the MOs forming the $\mathrm{C}=\mathrm{C}$ band are enhanced by the strong vibronic coupling with the symmetry breaking vibrational modes provoking the ring opening reaction. ${ }^{11,12}$

In this work, we found that the excitation to the 3p Rydberg states leads to the longer time for the ring-opening reaction than that to the $1^{1} \mathrm{~B}$ state. ${ }^{11,12,19}$ During sliding down to the $2^{1} \mathrm{~A}$ potential energy surface, the absorbed photon energy is redistributed among the vibrational modes in the isolated molecules and the molecule excited to the $3 p$ Rydberg states has more internal energy than that to the $1^{1} \mathrm{~B}$ state. Thus, it takes more time for the energy dissipation to reach the $\mathrm{CI}$ on the $2^{1} \mathrm{~A}$ potential energy surface compared with the excitation to the $1^{1} \mathrm{~B}$ state.

\section{Estimation of the ring-opening efficiency}

The quantum yield for the ring-opening reaction was estimated as follows: Fig. 4a shows that the band between 13 and $14 \mathrm{eV}$ was not recovered at $1000 \mathrm{fs}$, which should be due to cZc-HT formation. We focus on the band between 13 and $14 \mathrm{eV}$, because the intensity at $607 \mathrm{fs}$, when the ring was not fully opened, was still almost the same as at 154 fs and because it was less modified by the recovery of the $\mathrm{C}=\mathrm{C}$ band. The reduced area at $154 \mathrm{fs}$ is attributed to the depletion of the ground state of CHD. If the recovered portion at $1000 \mathrm{fs}$ is attributed totally to the ground state of $\mathrm{CHD}$, the quantum yield of $\mathrm{cZc}-\mathrm{HT}$ is estimated to be 0.46 . Here, the ionization cross section between 13 and $14 \mathrm{eV}$ of cZc-HT is assumed to be zero in this estimation. So 0.46 is the minimum quantum yield for the ring-opening reaction after the two-photon excitation to the FC states of the 3p Rydberg states. On the other hand, the efficiency in the solvent ${ }^{45}$ and in gas phase by TRPES ${ }^{16}$ upon $1^{1} \mathrm{~B}$ excitation were 0.4 and 0.3 , respectively. According to the theoretical work, ${ }^{46}$ the quantum efficiency upon $2^{1} \mathrm{~A}$ excitation is lower than that upon $1^{1} \mathrm{~B}$. Since the initial states are different in the present case, the efficiency is not comparable. We suspect that the excess energy by photoexcitation to $3 p$ Rydberg states promotes the ring opening deformation on the $2^{1} \mathrm{~A}$ potential energy surface.

\section{Conclusions}

TRPES using high harmonic pulses shed light on the detailed structural dynamics of the ring-opening processes of CHD by correlating the lower-lying MOs with particular spatial electron distributions predicted by quantum chemical calculation. Upon two-photon excitation to the FC state of $3 p$ Rydberg states, the electronic state slides to $2^{1} \mathrm{~A}$ with a time constant of $37 \pm 13 \mathrm{fs}$. On the $2{ }^{1} \mathrm{~A}$ potential energy surface, the ring structure might be deformed to $5 \mathrm{MR}$, where a CI with the ground state is located. Then, the ring-opening reaction to cZc-HT starts at $\sim 500$ fs and finishes at $\sim 800$ fs. The isomerization to cZt-HT or to tZt-HT proceeds upto $1.4 \mathrm{ps}$. The long-term measurement suggests further isomerization among HT isomers. 


\section{Acknowledgements}

This work is supported by CREST, JST. T. S. was also supported by KAKENHI (26600107, 15H03702, and 16K13854) and the Eno Science Foundation. T. T. was also supported by KAKENHI (26288001). Part of the calculations was performed on supercomputers at the Research Center for Computational Science, Okazaki, Japan.

\section{References}

1 C. M. Beaudry, J. P. Malerich and D. Trauner, Chem. Rev., 2005, 105, 4757-4778.

2 M. Irie, Chem. Rev., 2000, 100, 1683-1684.

3 R. B. Woodward and R. Hoffmann, Angew. Chem., Int. Ed. Engl., 1969, 8, 781853.

4 B. C. Arruda and R. J. Sension, Phys. Chem. Chem. Phys., 2014, 16, 4439-4455.

5 S. Deb and P. M. Weber, Annu. Rev. Phys. Chem., 2011, 62, 19-39.

6 H. J. C. Jacobs, J. W. J. Gielen and E. Havinga, Tetrahedron Lett., 1981, 22, 40134016.

7 M. Braun, W. Fuß, K. L. Kompa and J. Wolfrum, J. Photochem. Photobiol., A, 1991, 61, 15-26.

8 O. G. Dmitrenko, I. P. Terenetskayab and W. Reischl, J. Photochem. Photobiol., A, 1997, 104, 113-117.

9 S. A. Trushin, W. Fuß, T. Schikarski, W. E. Schmid and K. L. Kompa, J. Chem. Phys., 1997, 106, 9386.

10 W. Fuß, W. E. Schmid and S. A. Trushin, J. Chem. Phys., 2000, 112, 8347-8362.

11 N. Kuthirummal, F. M. Rudakov, C. L. Evans and P. M. Weber, J. Chem. Phys., 2006, 125, 133307.

12 K. Kosma, S. A. Trushin, W. Fuß and W. E. Schmid, Phys. Chem. Chem. Phys., 2009, 11, 172-181.

13 J. L. White, J. Kim, V. S. Petrovi and P. H. Bucksbaum, J. Chem. Phys., 2012, 136, 054303.

14 V. S. Petrović, M. Siano, J. L. White, N. Berrah, C. Bostedt, J. D. Bozek, D. Broege, M. Chalfin, R. N. Coffee, J. Cryan, L. Fang, J. P. Farrell, L. J. Frasinski, J. M. Glownia, M. Gühr, M. Hoener, D. M. P. Holland, J. Kim, J. P. Marangos, T. Martinez, B. K. McFarland, R. S. Minns, S. Miyabe, S. Schorb, R. J. Sension, L. S. Spector, R. Squibb, H. Tao, J. G. Underwood and P. H. Bucksbaum, Phys. Rev. Lett., 2012, 108, 253006.

15 V. S. Petrovic, S. Schorb, J. Kim, J. White, J. P. Cryan, J. M. Glownia, L. Zipp, D. Broege, S. Miyabe, H. Tao, T. Martinez and P. H. Bucksbaum, J. Chem. Phys., 2013, 139, 184309.

16 S. Adachi, M. Sato and T. Suzuki, J. Phys. Chem. Lett., 2015, 6, 343-346.

17 C. C. Pemberton, Y. Zhang, K. Saita, A. Kirrander and P. M. Weber, J. Phys. Chem. A, 2015, 119, 8832-8845.

18 C.-Y. Ruan, V. A. Lobastov, R. Srinivasan, B. M. Goodson, H. Ihee and A. H. Zewail, Proc. Natl. Acad. Sci. U. S. A., 2001, 98, 7117-7122.

19 M. P. Minitti, J. M. Budarz, A. Kirrander, J. S. Robinson, D. Ratner, T. J. Lane, D. Zhu, J. M. Glownia, M. Kozina, H. T. Lemke, M. Sikorski, Y. Feng, S. Nelson, K. Saita, B. Stankus, T. Northey, J. B. Hastings and P. M. Weber, Phys. Rev. Lett., 2015, 114, 255501. 
20 M. Merchán, L. Serrano-Andrés, L. S. Slater, B. O. Roos, R. McDiarmid and X. Xing, J. Phys. Chem. A, 1999, 103, 5468-5476.

21 A. Hofmann, L. Kurtz and R. d. Vivie-Riedle, Appl. Phys. B, 2000, 71, 391-396.

22 M. Garavelli, C. S. Page, P. Celani, M. Olivucci, W. E. Schmid, S. A. Trushin and W. Fuss, J. Phys. Chem. A, 2001, 105, 4458-4469.

23 H. Tamura, S. Nanbu, T. Ishida and H. Nakamura, J. Chem. Phys., 2006, 124, 084313.

24 A. Nenov, P. Kolle, M. A. Robb and R. d. Vivie-Riedle, J. Org. Chem., 2010, 75, 123-129.

25 T. Tsuneda, J.-W. Song, S. Suzuki and K. Hirao, J. Chem. Phys., 2010, 133, 174101.

26 L. Nugent-Glandorf, M. Scheer, D. A. Samuels, A. M. Mulhisen, E. R. Grant, X. Yang, V. M. Bierbaum and S. R. Leone, Phys. Rev. Lett., 2001, 87, 193002.

27 P. Wernet, M. Odelius, K. Godehusen, J. Gaudin, O. Schwarzkopf and W. Eberhardt, Phys. Rev. Lett., 2009, 103, 013001.

28 P. Wernet, Phys. Chem. Chem. Phys., 2011, 13, 16941-16954.

29 M. Fushitani, A. Matsuda and A. Hishikawa, Opt. Express, 2011, 19, 9600-9606.

30 A. Makida, H. Igarashi, T. Fujiwara, T. Sekikawa, Y. Harabuchi and T. Taketsugu, J. Phys. Chem. Lett., 2014, 5, 1760-1765.

31 Handbook of HeI Photoelectron Spectra of Fundamental Organic Molecules : Ionization Energies, Ab Initio Assignments, and Valence Electronic Structure for 200 Molecules, ed. K. Kimura, Japan Scientific Societies Press, Tokyo, 1981.

32 R. Iikubo, T. Fujiwara, T. Sekikawa, Y. Harabuchi, S. Satoh, T. Taketsugu and Y. Kayanuma, J. Phys. Chem. Lett., 2015, 6, 2463-2468.

33 P. Villoresi, Appl. Opt., 1999, 38, 6040-6049.

34 L. Poletto, P. Villoresi, E. Benedetti, F. Ferrari, S. Stagira, G. Sansone and M. Nisoli, Opt. Lett., 2007, 32, 2897-2899.

35 H. Igarashi, A. Makida, M. Ito and T. Sekikawa, Opt. Express, 2012, 20, 37253732.

36 H. Igarashi, A. Makida and T. Sekikawa, Opt. Express, 2013, 21, 20632-20640.

37 M. Ito, Y. Kataoka, T. Okamoto, M. Yamashita and T. Sekikawa, Opt. Express, 2010, 18, 6071-6078.

38 T. E. Glover, R. W. Schoenlein, A. H. Chin and C. V. Shank, Phys. Rev. Lett., 1996, 76, 2468-2471.

39 T. Sekikawa, T. Katsura, S. Miura and S. Watanabe, Phys. Rev. Lett., 2002, 88, 193902.

40 M. S. Gordon and M. W. Schmidt, in Theory and Applications of ComputationalChemistry, the First Forty Years, ed. C. E. Dykstra, G. Frenking, K. S. Kim and G. E. Scuseria, Elsevier, Amsterdam, 2005, ch. 41, pp. 1167-1189.

41 T. H. Dunning Jr., J. Chem. Phys., 1970, 53, 2823.

42 H.-J. Werner, P. J. Knowles, G. Knizia, F. R. Manby, M. Schütz, P. Celani, T. Korona, R. Lindh, A. Mitrushenkov, G. Rauhut, K. R. Shamasundar, T. B. Adler, R. D. Amos, A. Bernhardsson, A. Berning, D. L. Cooper, M. J. O. Deegan, A. J. Dobbyn, F. Eckert, E. Goll, C. Hampel, A. Hesselmann, G. Hetzer, T. Hrenar, G. Jansen, C. Köppl, Y. Liu, A. W. Lloyd, R. A. Mata, A. J. May, S. J. McNicholas, W. Meyer, M. E. Mura, A. Nicklass, D. P. O'Neill, P. Palmieri,D. Peng, K. Pflüger, R. Pitzer, M. Reiher, T. Shiozaki, H. Stoll, A. J. Stone, R. Tarroni, T. Thorsteinsson and M. Wang, Molpro, Version 2015.1, a package of ab initio programs, 2015, see http://www.molpro.net. 
43 R. McDiarmid, A. Sabljić and J. P. Doering, J. Chem. Phys., 1985, 83, 2147-2152. 44 P. Kruit and F. H. Read, J. Phys. E: Sci. Instrum., 1983, 16, 313-324.

45 S. H. Pullen, N. A. Anderson, L. A. Walker and R. J. Sension, J. Chem. Phys., 1998, 108, 556-563.

46 A. Ohta, O. Kobayashi, S. O. Danielache and S. Nanbu, Chem. Phys., 2015, 459, $45-53$. 